\title{
Effects on the Human Body of a Dietary Supplement Containing L-Carnitine and Garcinia cambogia Extract: A Study using Double-blind Tests
}

\author{
Yoshikazu Yonei ${ }^{1, *}$, Yoko Takahashi ${ }^{1}$, Sawako Hibino ${ }^{1}$, Miwako Watanabe ${ }^{2}$, \\ and Toshito Yoshioka ${ }^{3}$ \\ ${ }^{1}$ Anti-Aging Medical Research Center, Doshisha University, Kyoto 610-0394, Japan \\ ${ }^{2}$ Mareesia Garden Clinic, Tokyo 163-1011, Japan \\ ${ }^{3}$ Waseda University Graduate School of Commerce, Tokyo 165-8050, Japan
}

Received 13 March, 2006; Accepted 20 February, 2007

\begin{abstract}
Summary The effect of a dietary supplement with L-carnitine $(600 \mathrm{mg} / \mathrm{day})$ and Garcinia cambogia extract $(500 \mathrm{mg} /$ day as hydroxycitric acid) as main ingredients was studied in 35 healthy volunteers $\{48.3 \pm 6.9$ years, body mass index $(B M I): 26.3 \pm 1.7\}$ in a double-blind test (18 subjects in the Test Group and 17 in the Control Group). The yearly examination includes the standard yearly medical tests done in Japan, tests for assessing hormonal age, and a survey for assessing physical and mental fitness of the subjects, called the Anti-Aging QOL Common Questionnaire (AAQol). Use of this supplement significantly improved the level of lipid peroxides $(-12.8 \%)$ in the blood as well as physical symptoms such as "tired eyes," "blurry eyes," "muscle pain/stiffness," "early satiety," "epigastralgia," "dizziness," "arthralgia" and "easily breaking into a sweat." The Control Group showed a significantly favorable improvement rate, especially for "dizziness." On the other hand, groups of subjects using the test compounds saw a significant rise in total cholesterol (4.5\%), fasting blood sugar $(4.1 \%)$ and HbA1c (3.4\%). Our findings suggest that the consumption of the supplement can reduce the oxidative damage; however, the effect on QOL was equivocal. Garcinia cambogia extract did not show dietary efficacy.
\end{abstract}

Key Words: exercise, total cholesterol, body fat, insulin, 8-OHdG

\section{Introduction}

The objective of anti-aging medicine is to live as long as possible in good health. It is medical treatment that not only prolongs human life, but also enables a person to live a healthy and happy life, maintain quality of life (QOL), and reduce the physical and mental deterioration of aging [1]. Medically, anti-aging medicine is classified as prophylactic

\footnotetext{
*To whom correspondence should be addressed

Tel: +81-75-391-5644 Fax: +81-3-5730-1447

E-mail: yyonei@mail.doshisha.ac.jp
}

medicine. Checkups and screenings for assessing the degree of aging are offered in Japan; a range of treatment options is available including diet, exercise, other lifestyle therapies, and drug therapies including nutritional supplement therapies and hormone replacement therapies.

Terms such as integrated medicine, alternative healthcare, Chinese herbal medicine, acupuncture therapy, and antiaging medicine have been discussed in recent years, and numerous treatment methods are being proposed that differ from conventional Western medicine. Although numerous reports cite the efficacy of such treatment methods, the parameters used differ from one study to another. General physicians are currently left with the basic question, "To 
what degree are these treatments effective, and for what types of symptoms and diseases?"

The authors have used common parameters to investigate and study (a) the assessments of general physical examinations, (b) the physical and psychological influence as well as safety of dietary supplements, health foods, medical devices, exercise equipment, and cosmetics, and (c) obesity [2-4]. Our findings are shedding more light on the efficacies, limitations, and the range of effects of these products. Amino acids, in particular, exert a variety of influences on the body, and we have therefore conducted studies on health foods containing amino acids. In this study, we have focused on a food product for which one manufacturer has made health claims; the product contains L-carnitine, a type of amino acid, and Garcinia cambogia extract (65\% hydroxycitric acid) manufactured by Nurex Corporation, Osaka; hereinafter referred to as "the Product". We studied, using healthy middle-aged and older subjects, the effects of the Product on the body as a double-blind, non-cross-matching test. We also studied parameters that are deemed especially important to anti-aging medicine, for the purpose of determining whether or not the use of the Product enhances QOL in people aged in their 40 s to 60 s.

\section{Experimental Procedures}

\section{Subjects}

The subjects of our study were healthy individuals aged in their $40 \mathrm{~s}$ to $60 \mathrm{~s}$, with a BMI of 24 or higher, who had provided us with their written consent to participate in this study. The following individuals were excluded:

- those taking hormones, anti-osteoporosis drugs, and other medications

- those with diabetes, marked hepatic dysfunction, or other chronic diseases

- those with severe liver, kidney, heart, and/or blood diseases and other complications

- other individuals judged by a physician as unfit to participate in the study

Prior to the start of the test, we provided potential test subjects a thorough explanation not only of the duration, place, content, and methods of the test, but also on the benefits anticipated to be gained from participating in the study as well as disadvantages that may occur. The test subjects were assured that participation was completely voluntary, and that they would not suffer any disadvantages whatsoever, if they decided mid-way to withdraw from the testing, for any reason. The method of taking the Product was explained to all test participants by Nurex Corporation, either in writing or by holding an explanatory meeting.

\section{Design}

The period of the test was determined to be 8 weeks from
October 3 to November 28, 2004. Before and after the test, the following items were measured at the Mareesia Garden Clinic, Tokyo.

- physical measurements: Height $(\mathrm{cm})$, weight $(\mathrm{kg})$, body fat percentage, systolic and diastolic blood pressures and others

- blood tests: Platelet count $\left(10^{4} / \mu \mathrm{l}\right)$, erythrocyte count

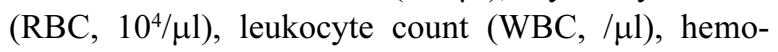
globin content (HGB, g/dl), hematocrit (HCT, \%), glutamate-oxaloacetate transaminase, glutamatepyruvate transaminase (GOT, GPT, IU/l), $\gamma$-glutamyl transpeptase ( $\gamma$-GTP, IU/1), alkaline phosphatase (ALP, IU/l), creatinine phosphokinase (CPK, mU/ml), Creactive protein $(\mathrm{CRP}, \mathrm{mg} / \mathrm{dl})$, blood urea nitrogen (BUN, mg/dl), blood creatinine $(\mathrm{Cr}, \mathrm{mg} / \mathrm{dl})$, uric acid (UA, mg/dl), total cholesterol (T-Chol, mg/dl), HDLcholesterol (HDL-C, mg/dl), LDL-cholesterol (LDL$\mathrm{C}, \mathrm{mg} / \mathrm{dl}$ ), triglycerides ( $\mathrm{TG}, \mathrm{mg} / \mathrm{dl})$, fasting blood sugar (FBS, mg/dl), glycohemoglobin A1C (HbA1c), lactic acid (mg/dl), cortisol, dehydroepiandrosterone sulfate (DHEA-s), IGF-I, insulin, lipid peroxides (LPO, $\mathrm{nmol} / \mathrm{ml})$, electrolytes: sodium $(\mathrm{Na}, \mathrm{mEq} / \mathrm{l})$, potassium $\left(\mathrm{K}^{+}, \mathrm{mEq} / \mathrm{l}\right)$, and chloride $\left(\mathrm{Cl}^{-}, \mathrm{mEq} / \mathrm{l}\right)$.

- urine tests: Urinary 8-hydroxy-deoxyguanosine (8$\mathrm{OHdG}$ ) and urinary isoprostane

- observations on improvement of QOL using a common questionnaire sheet

If circumstances such as the following occurred, necessitating discontinuation of the test, the test was discontinued immediately, following the decision of the physician in charge of the test, and adequate treatment was provided.

- if other serious diseases are seen to have developed

- if existing symptoms (including complications) worsened

- if a physician determines that continuation of the test is impossible for other reasons

Our present study targeted 35 healthy individuals (18 men and 17 women) $\{48.3 \pm 6.9$ years, body mass index (BMI): $26.3 \pm 1.7\}$ who were randomly divided into the Test Group and the Control Group. At least 6 cases in each group are necessary for detection of the significant difference in any of more than 50 items in the Anti-Aging QOL Common Questionnaire (AAQol) [2-4], and the number should be doubled or tripled for the classified analysis according to 2 or 3 classes. There were 18 individuals in the Test Group (age: $48.6 \pm 6.0$ years, height: $163.1 \pm 7.9 \mathrm{~cm}$; weight: $69.9 \pm 7.2 \mathrm{~kg}$, and BMI: $26.3 \pm 1.7$; mean $\pm \mathrm{SD}$ ), and 17 in the Control Group (age: $48.0 \pm 7.9$ years, height: $163.5 \pm$ $11.2 \mathrm{~cm}$, weight: $66.5 \pm 11.9 \mathrm{~kg}$, and BMI: $24.7 \pm 2.9$; mean \pm SD). The Test Group took 6 doses of the Product, including L-carnitine (600 mg/day) and Garcinia cambogia extract $(500 \mathrm{mg} /$ day as hydroxycitric acid) for 8 weeks, while the Control Group took 6 doses of a placebo drug for 
8 weeks. The method of taking these test drugs was explained by a nutritionist to all of the test subjects.

The Product is manufactured by Nurex Corporation of Osaka Prefecture, Japan, and has the following composition.

Raw materials:

L-carnitine, L-tartrate, Garcinia cambogia extract, corn starch, edible purified oil-processed fat and oil (hydrogenated oil), gelatin, caramel coloring, calcium pantothenate, vitamin $\mathrm{B}_{1}$. Nutritional ingredients (for every 3 doses of the Product): calories: $4.58 \mathrm{kcal}$, protein: $0.38 \mathrm{~g}$, fat: $0.06 \mathrm{~g}$, carbohydrates: $0.61 \mathrm{~g}$, sodium: $1.26 \mathrm{mg}$, vitamin $\mathrm{B}_{1}$ : $1.2 \mathrm{mg}$, pantothenic acid: $10 \mathrm{mg}$, L-carnitine: $300 \mathrm{mg}$, hydroxycitric acid (Garcinia cambogia extract): $250 \mathrm{mg}$. The amounts of vitamin $\mathrm{B}_{1}$ and pantothenic acid are subtle compared with those of daily dietary intake.

As indicators for the degree of oxidation stress, we used serum lipid peroxide, 8-OHdG (8-hydroxy-2' deoxyguanosine), and isoprostane (8-isoprostane F-2 $\alpha$ ) [5-7]. These indicators were measured at the Japan Institute for the Control of Aging. Moreover, the $8-\mathrm{OHdG}$, isoprostane, and creatinine contained in the first urine in the early morning were measured to calculate the rate of $8-\mathrm{OHdG} / \mathrm{CRE}$. isoprostane production and the creatinine correction volume (8-OHdG/CRE-isoprostane/CRE), based on the volume of urine and urine collection period (the time from the last urination of the previous night to the first urination the following morning).

Subjective symptoms were divided into physical and mental symptoms and evaluated on a scale of 1 to 5 , using the Anti-Aging QOL Common Questionnaire (AAQol) [2-4].

\section{Statistical analysis}

A statistical analysis of the results was performed using the paired t-test, non-paired t-test, or non-parametric test (Wilcoxon's signed rank test). The relationship between each parameter was tested using Pearson's correlation coefficient. A value of $\mathrm{p}<0.05$ was considered to indicate a statistically significant change. Dr.SPSSII, a software program (manufactured by SPSS Inc.) was used in this study.

\section{Results}

\section{Test results}

Tables 1 and 2 show the physical measurement values. The Test Group showed no significant changes in height, weight, body water content, muscle volume, lean muscle mass, skeletal muscle volume, body fat volume, BMI, body fat percentage, waist-hip ratio, basal metabolism, or blood pressure. The Control Group, on the other hand, showed slightly significant changes in body water content $(2.1 \%$, $p=0.002)$, muscle volume $(2.0 \%, p=0.002)$, lean muscle mass $(2.0 \%, p=0.002)$ and basal metabolism $(1.4 \%$, $p=0.002)$. We compared the rates before and after the test between the Test and the Control Groups and saw no significant intra-group differences in any of the items.

In the general laboratory tests (Tables 3 and 4), the Test Group showed significant changes in their total cholesterol $(4.5 \%)$ from $214.0 \pm 31.1$ to $223.7 \pm 31.44 \quad(p=0.046)$, fasting blood sugar $(4.1 \%)$ from $91.9 \pm 10.6$ to $95.7 \pm 7.5$ $(p=0.016)$, and HbAlc (3.4\%) from $4.73 \pm 0.43$ to $4.89 \pm 0.39(p=0.000)$. Significant fluctuations were also seen in $\mathrm{MCV}(1.4 \%)$ from $93.5 \pm 3.3$ to $94.8 \pm 3.2$ $(p=0.000)$ and $\mathrm{MCH}(1.3 \%)$ from $30.6 \pm 1.5$ to $31.0 \pm 1.4$ $(p=0.000)$, but they were slight changes within the range of $1.4 \%$. The Control Group showed significant variations in the leukocyte count $(-10.9 \%)$ from $7244.0 \pm 2665.9$ to $6456.7 \pm 2120.0 \quad(p=0.020)$ and HbAlc $(2.9 \%)$ from $4.89 \pm 0.40$ to $5.03 \pm 0.43(p=0.001)$. Although a significant fluctuation was seen in MCV $(1.0 \%)$ from $92.6 \pm 5.6$ to $93.5 \pm 5.7(p=0.038)$, the change was only $1.0 \%$. Intragroup analysis showed no significant differences between the two groups.

The Test Group showed no significant variations in lactic acid, insulin, IGF-I, cortisol, or DHEA-s (Tables 3 and 4). The Control Group, meanwhile, showed a significant variation in IGF-I $(-8.8 \%)$ from $197.9 \pm 68.9$ to $180.6 \pm 60.4$ $(p=0.007)$. An intra-group analysis showed no significant differences between the two groups.

Regarding oxidation stress markers (Tables 3 and 4), the Test Group showed a significant reduction in blood lipid peroxide $(-12.8 \%)$ from $0.39 \pm 0.07$ to $0.34 \pm 0.08$ ( $p=$ 0.001 ) but no significant changes in the speed of urinary 8 -OHdG formation, or the speed of isoprostane formation, 8-OHdG/CRE, or isoprostane/CRE. The Control Group, on the other hand, showed no significant changes in lipid peroxides, and no significant variations in other urinary markers. An intra-group analysis showed no significant differences between the two groups.

Tables 5-8 show the results of the analysis on the AntiAging QOL Common Questionnaire (AAQol). Regarding physical symptoms, the Test Group showed significant improvements in scores of the following 8 out of 30 items: "tired eyes" ( $p=0.015)$, "blurry eyes" $(p=0.035)$, "muscle pain/stiffness" ( $p=0.017)$, "early satiety" $(p=0.042)$, “epigastralgia" ( $p=0.010)$, “dizziness" ( $p=0.003)$, "arthralgia" $(p=0.037)$, and "easily breaking into a sweat" $(p=0.026)$. The Control Group did not show improvements in any of the scores. Intra-group analysis showed a significant difference between the two groups in terms of changes in the score for "dizziness" ( $p=0.008)$.

As for mental symptoms, the Test Group showed no items (of the 21 items) whose scores had improved. The Control Group showed a significant improvement in the score for a single item: "sleeping disturbance" $(p=0.019)$. An intragroup analysis showed no significant differences between the two groups. 
Table 1. Physical measurements of Test Group

\begin{tabular}{|c|c|c|c|c|c|}
\hline & Unit & Before & 8 weeks later & $\begin{array}{c}P \text { value vs } \\
\text { "before" value }\end{array}$ & $\begin{array}{c}P \text { value vs } \\
\text { Control Group }\end{array}$ \\
\hline Height & $\mathrm{kg}$ & $163.1 \pm 7.9$ & $163.2 \pm 7.8$ & 0.348 & 0.846 \\
\hline Weight & $\mathrm{kg} / \mathrm{m}^{2}$ & $69.9 \pm 7.2$ & $70.1 \pm 7.0$ & 0.439 & 0.964 \\
\hline Total body water & $\mathrm{L}$ & $33.8 \pm 5.9$ & $34.4 \pm 5.5$ & 0.083 & 0.983 \\
\hline Muscle volume & $\mathrm{cm}$ & $46.2 \pm 8.0$ & $47.0 \pm 7.5$ & 0.079 & 0.983 \\
\hline Lean body weight & $\mathrm{kg}$ & $48.9 \pm 8.4$ & $49.8 \pm 7.8$ & 0.084 & 0.984 \\
\hline Skeletal muscle volume & $\mathrm{kg}$ & $26.4 \pm 5.3$ & $26.5 \pm 4.9$ & 0.667 & 0.597 \\
\hline Body fat amount & $\mathrm{kg}$ & $21.0 \pm 3.9$ & $20.5 \pm 3.7$ & 0.238 & 0.977 \\
\hline BMI & & $26.3 \pm 1.7$ & $26.3 \pm 1.7$ & 0.643 & 0.999 \\
\hline Body fat percentage & $\%$ & $29.8 \pm 6.5$ & $29.4 \pm 6.0$ & 0.652 & 0.614 \\
\hline Waist/hip ratio & & $0.92 \pm 0.04$ & $0.91 \pm 0.04$ & 0.331 & 0.797 \\
\hline Basal metabolism & kcal & $1315.8 \pm 175.2$ & $1331.3 \pm 166.9$ & 0.094 & 0.965 \\
\hline Systolic pressure & $\mathrm{mmHg}$ & $126.9 \pm 12.0$ & $125.9 \pm 12.1$ & 0.608 & 0.665 \\
\hline Diastolic pressure & $\mathrm{mmHg}$ & $83.4 \pm 7.7$ & $81.6 \pm 8.2$ & 0.275 & 0.972 \\
\hline
\end{tabular}

$n=18$, mean $\pm \mathrm{SD}$, paired $t$ test or Mann-Whitney $U$ test

Table 2. Physical measurements of Control Group

\begin{tabular}{llcccc}
\hline & Unit & Before & 8 weeks later & $\begin{array}{c}P \text { value vs } \\
\text { "before" value }\end{array}$ & $\begin{array}{c}P \text { value vs } \\
\text { Test Group }\end{array}$ \\
\hline Height & $\mathrm{kg}$ & $163.6 \pm 11.2$ & $163.5 \pm 11.2$ & 0.924 & 0.846 \\
Weight & $\mathrm{kg} / \mathrm{m}^{2}$ & $66.5 \pm 11.9$ & $66.6 \pm 12.2$ & 0.714 & 0.964 \\
Total body water & $\mathrm{L}$ & $33.8 \pm 7.8$ & $34.5 \pm 7.8$ & 0.002 & 0.983 \\
Muscle volume & $\mathrm{cm}$ & $46.1 \pm 10.6$ & $47.0 \pm 10.6$ & 0.002 & 0.983 \\
Lean body weight & $\mathrm{kg}$ & $48.8 \pm 11.1$ & $49.8 \pm 11.1$ & 0.002 & 0.984 \\
Skeletal muscle volume & $\mathrm{kg}$ & $26.3 \pm 7.2$ & $26.7 \pm 7.0$ & 0.062 & 0.597 \\
Body fat amount & $\mathrm{kg}$ & $17.6 \pm 4.5$ & $17.3 \pm 4.8$ & 0.209 & 0.977 \\
BMI & & $24.7 \pm 2.9$ & $24.7 \pm 3.0$ & 0.748 & 0.999 \\
Body fat percentage & $\%$ & $27.0 \pm 6.5$ & $26.1 \pm 6.7$ & 0.082 & 0.614 \\
Waist/hip ratio & & $0.89 \pm 0.04$ & $0.89 \pm 0.04$ & 0.135 & 0.797 \\
Basal metabolism & $\mathrm{kcal}$ & $1325.8 \pm 243.2$ & $1344.3 \pm 243.7$ & 0.002 & 0.965 \\
Systolic pressure & $\mathrm{mmHg}$ & $132.3 \pm 18.8$ & $126.7 \pm 15.8$ & 0.210 & 0.665 \\
Diastolic pressure & $\mathrm{mmHg}$ & $86.1 \pm 13.8$ & $83.0 \pm 13.5$ & 0.316 & 0.972 \\
\hline
\end{tabular}

$n=17$, mean $\pm \mathrm{SD}$, paired $t$ test or Mann-Whitney $U$ test

\section{Variations associated with gender}

A factoral correlational analysis was performed by gender and for each item. Results of physical measurements showed higher values in men for height, weight, body water content, muscle volume, lean muscle mass, skeletal muscle volume and basal metabolism. Women had a significantly higher percentage of body fat. Regarding the lifestyles of subjects, men showed a significantly higher use of alcoholic drinks and cigarettes.

Regarding physical symptoms, slightly more men than women suffered "diarrhea" and "lumbago" $(p<0.05)$, while more women than men suffered "excessive sensitivity to cold" $(p<0.01)$. No gender differences were seen in mental symptoms.

Regarding general laboratory test items, men showed significantly higher values than women in their erythrocyte 
Table 3. Clinical tests of Test Group

\begin{tabular}{|c|c|c|c|c|c|}
\hline & Unit & Before & 8 weeks later & $\begin{array}{l}P \text { value vs } \\
\text { "before" value }\end{array}$ & $\begin{array}{c}P \text { value vs } \\
\text { Control Group }\end{array}$ \\
\hline Leukocyte Count (WBC) & $/ \mu 1$ & $6051.1 \pm 1506.1$ & $6008.9 \pm 1717.5$ & 0.841 & 0.335 \\
\hline Erythrocyte Count (RBC) & $10^{4} / \mu 1$ & $471.9 \pm 33.6$ & $471.9 \pm 41.6$ & & 0.865 \\
\hline Hemoglobin Content (HGB) & $\mathrm{g} / \mathrm{dl}$ & $14.5 \pm 1.3$ & $14.6 \pm 1.4$ & 0.149 & 0.497 \\
\hline Hematocrit (HCT) & $\%$ & $44.1 \pm 3.3$ & $44.7 \pm 3.9$ & 0.183 & 0.741 \\
\hline Mean Corpuscular Volume (MCV) & $\mathrm{fl}$ & $93.5 \pm 3.3$ & $94.8 \pm 3.2$ & 0.000 & 0.736 \\
\hline Mean Corpuscular Hemoglobin (MCH) & pg & $30.6 \pm 1.5$ & $31.0 \pm 1.4$ & 0.000 & 0.270 \\
\hline $\begin{array}{l}\text { Mean Corpuscular Hemoglobin } \\
\text { Concentration (MCHC) }\end{array}$ & $\%$ & $32.7 \pm 0.9$ & $32.7 \pm 0.9$ & & 0.568 \\
\hline Platelet count & $10^{4} / \mu 1$ & $22.5 \pm 3.3$ & $22.9 \pm 4.1$ & 0.470 & 0.867 \\
\hline $\begin{array}{l}\text { Glutamate Oxaloacetate Transaminase } \\
\text { (GOT) }\end{array}$ & $\mathrm{U} / 1$ & $24.6 \pm 11.5$ & $23.7 \pm 9.9$ & 0.368 & 0.974 \\
\hline $\begin{array}{l}\text { Glutamate Pyruvate } \\
\text { Transaminase (GPT) }\end{array}$ & $\mathrm{U} / 1$ & $32.2 \pm 30.2$ & $30.2 \pm 25.4$ & 0.401 & 0.992 \\
\hline Alkaline phosphatase (ALP) & $\mathrm{IU} / 1$ & $238.7 \pm 66.5$ & $227.4 \pm 56.6$ & 0.161 & 0.889 \\
\hline$\gamma$-glutamyl transpeptase $(\gamma$-GTP) & $\mathrm{IU} / 1$ & $59.3 \pm 49.8$ & $54.2 \pm 47.1$ & 0.236 & 0.790 \\
\hline Blood Creatinine $(\mathrm{Cr})$ & $\mathrm{mg} / \mathrm{dl}$ & $0.68 \pm 0.12$ & $0.69 \pm 0.13$ & 0.300 & 0.962 \\
\hline Blood Urea Nitrogen (BUN) & $\mathrm{mg} / \mathrm{dl}$ & $13.4 \pm 2.8$ & $12.7 \pm 2.5$ & 0.274 & 0.587 \\
\hline Uric acid (UA) & $\mathrm{mg} / \mathrm{dl}$ & $5.69 \pm 1.42$ & $5.51 \pm 1.26$ & 0.235 & 0.570 \\
\hline Creatine Phosphokinase (CPK) & $\mathrm{mU} / \mathrm{ml}$ & $126.4 \pm 58.1$ & $123.9 \pm 76.6$ & 0.826 & 0.429 \\
\hline Total cholesterol & $\mathrm{mg} / \mathrm{dl}$ & $214.0 \pm 31.1$ & $223.7 \pm 31.4$ & 0.046 & 0.887 \\
\hline HDL-cholesterol & $\mathrm{mg} / \mathrm{dl}$ & $52.8 \pm 12.4$ & $54.4 \pm 12.7$ & 0.182 & 0.525 \\
\hline LDL-cholesterol & $\mathrm{mg} / \mathrm{dl}$ & $135.7 \pm 29.9$ & $142.3 \pm 26.8$ & 0.091 & 0.691 \\
\hline Triglycerides (TG) & $\mathrm{mg} / \mathrm{dl}$ & $144.0 \pm 78.1$ & $136.9 \pm 68.8$ & 0.546 & 0.905 \\
\hline $\mathrm{Na}$ & $\mathrm{mEq} / 1$ & $142.8 \pm 1.5$ & $143.2 \pm 1.1$ & 0.130 & 0.983 \\
\hline $\mathrm{K}$ & $\mathrm{mEq} / 1$ & $4.19 \pm 0.37$ & $4.16 \pm 0.28$ & 0.622 & 0.882 \\
\hline $\mathrm{Cl}$ & $\mathrm{mEq} / 1$ & $104.9 \pm 2.1$ & $104.8 \pm 1.7$ & 0.915 & 0.905 \\
\hline Fasting Blood Sugar (FBS) & $\mathrm{mg} / \mathrm{dl}$ & $91.9 \pm 10.6$ & $95.7 \pm 7.5$ & 0.016 & 0.615 \\
\hline Glycohemoglobin A1C (HbA1c) & $\%$ & $4.73 \pm 0.43$ & $4.89 \pm 0.39$ & 0.000 & 0.799 \\
\hline C-Reactive Protein (CRP) & $\mathrm{mg} / \mathrm{dl}$ & $0.22 \pm 0.15$ & $0.21 \pm 0.14$ & 0.870 & 0.685 \\
\hline Lactic acid & $\mathrm{mg} / \mathrm{dl}$ & $9.54 \pm 5.83$ & $8.52 \pm 3.17$ & 0.279 & 0.471 \\
\hline Lipid peroxide (LPO) & $\mathrm{nmol} / \mathrm{ml}$ & $0.39 \pm 0.07$ & $0.34 \pm 0.08$ & 0.001 & 0.802 \\
\hline Insulin & $\mu \mathrm{U} / \mathrm{ml}$ & $7.56 \pm 4.12$ & $8.33 \pm 3.87$ & 0.364 & 0.648 \\
\hline IGF-I & $\mathrm{ng} / \mathrm{ml}$ & $185.3 \pm 42.9$ & $175.1 \pm 31.3$ & 0.160 & 0.636 \\
\hline Cortisol & $\mu \mathrm{g} / \mathrm{dl}$ & $11.4 \pm 5.0$ & $9.4 \pm 4.0$ & 0.071 & 0.562 \\
\hline $\begin{array}{l}\text { Dehydroepiandrosterone } \\
\text { Sulfate (DHEA-s) }\end{array}$ & $\mathrm{ng} / \mathrm{ml}$ & $1600.1 \pm 792.2$ & $1589.1 \pm 979.9$ & 0.928 & 0.993 \\
\hline $\begin{array}{l}\text { 8-hydroxy-deoxyguanosine } \\
\text { (8-OHdG) generation }\end{array}$ & $\mathrm{ng} / \mathrm{kg} / \mathrm{hr}$ & $8.78 \pm 5.2$ & $7.46 \pm 4.63$ & 0.186 & 0.549 \\
\hline Isoprostane generation & $\mathrm{ng} / \mathrm{kg} / \mathrm{hr}$ & $145.2 \pm 73.8$ & $114.4 \pm 69.6$ & 0.060 & 0.427 \\
\hline 8-OHdG/CRE & $\mathrm{ng} / \mathrm{mgCRE}$ & $126.0 \pm 65.3$ & $126.5 \pm 90.2$ & 0.488 & 0.353 \\
\hline Isoprostane/CRE & $\mathrm{ng} / \mathrm{mgCRE}$ & $1977.2 \pm 848.0$ & $1631.5 \pm 635.9$ & 0.054 & 0.338 \\
\hline
\end{tabular}

$n=18$, mean $\pm \mathrm{SD}$, paired $t$ test or Mann-Whitney $U$ test 
Table 4. Clinical tests of Control Group

\begin{tabular}{|c|c|c|c|c|c|}
\hline & Unit & Before & 8 weeks later & $\begin{array}{l}P \text { value vs } \\
\text { "before" value }\end{array}$ & $\begin{array}{l}P \text { value vs } \\
\text { Test Group }\end{array}$ \\
\hline Leukocyte Count (WBC) & $/ \mu 1$ & $7244.0 \pm 2665.9$ & $6456.7 \pm 2120.0$ & 0.020 & 0.335 \\
\hline Erythrocyte Count (RBC) & $10^{4} / \mu 1$ & $468.6 \pm 40.0$ & $464.7 \pm 42.0$ & 0.477 & 0.865 \\
\hline Hemoglobin Content (HGB) & $\mathrm{g} / \mathrm{dl}$ & $14.2 \pm 1.6$ & $14.2 \pm 1.7$ & 0.768 & 0.497 \\
\hline Hematocrit (HCT) & $\%$ & $43.3 \pm 3.7$ & $43.4 \pm 3.8$ & 0.863 & 0.741 \\
\hline Mean Corpuscular Volume (MCV) & $\mathrm{fl}$ & $92.6 \pm 5.6$ & $93.5 \pm 5.7$ & 0.038 & 0.736 \\
\hline Mean Corpuscular Hemoglobin (MCH) & pg & $30.4 \pm 2.6$ & $30.5 \pm 2.7$ & 0.293 & 0.270 \\
\hline $\begin{array}{l}\text { Mean Corpuscular Hemoglobin } \\
\text { Concentration (MCHC) }\end{array}$ & $\%$ & $32.8 \pm 1.3$ & $32.6 \pm 1.3$ & 0.309 & 0.568 \\
\hline Platelet count & $10^{4} / \mu \mathrm{l}$ & $26.4 \pm 4.5$ & $26.5 \pm 5.6$ & 0.810 & 0.867 \\
\hline $\begin{array}{l}\text { Glutamate Oxaloacetate Transaminase } \\
\text { (GOT) }\end{array}$ & $\mathrm{U} / 1$ & $23.4 \pm 5.3$ & $22.3 \pm 5.5$ & 0.408 & 0.974 \\
\hline $\begin{array}{l}\text { Glutamate Pyruvate } \\
\text { Transaminase (GPT) }\end{array}$ & $\mathrm{U} / 1$ & $27.3 \pm 11.3$ & $26.3 \pm 11.0$ & 0.618 & 0.992 \\
\hline Alkaline phosphatase (ALP) & $\mathrm{U} / 1$ & $251.3 \pm 71.2$ & $244.0 \pm 63.8$ & 0.443 & 0.889 \\
\hline$\gamma$-glutamyl transpeptase $(\gamma$-GTP) & $\mathrm{U} / 1$ & $49.1 \pm 40.2$ & $50.7 \pm 47.7$ & 0.736 & 0.790 \\
\hline Blood Creatinine $(\mathrm{Cr})$ & $\mathrm{mg} / \mathrm{dl}$ & $0.68 \pm 0.15$ & $0.69 \pm 0.15$ & 0.241 & 0.962 \\
\hline Blood Urea Nitrogen (BUN) & $\mathrm{mg} / \mathrm{dl}$ & $14.3 \pm 3.7$ & $14.2 \pm 2.6$ & 0.923 & 0.587 \\
\hline Uric acid (UA) & $\mathrm{mg} / \mathrm{dl}$ & $5.59 \pm 1.26$ & $5.64 \pm 1.14$ & 0.799 & 0.570 \\
\hline Creatine Phosphokinase (CPK) & $\mathrm{mg} / \mathrm{dl}$ & $138.6 \pm 91.0$ & $183.9 \pm 251.4$ & 0.327 & 0.429 \\
\hline Total cholesterol & $\mathrm{mg} / \mathrm{dl}$ & $224.6 \pm 47.2$ & $230.5 \pm 50.8$ & 0.346 & 0.887 \\
\hline HDL-cholesterol & $\mathrm{mg} / \mathrm{dl}$ & $51.5 \pm 7.8$ & $55.6 \pm 11.2$ & 0.069 & 0.525 \\
\hline LDL-cholesterol & $\mathrm{mg} / \mathrm{dl}$ & $147.6 \pm 46.3$ & $148.1 \pm 43.7$ & 0.910 & 0.691 \\
\hline Triglycerides (TG) & $\mathrm{mg} / \mathrm{dl}$ & $173.4 \pm 147.7$ & $132.3 \pm 57.7$ & 0.259 & 0.905 \\
\hline $\mathrm{Na}$ & $\mathrm{mEq} / \mathrm{l}$ & $142.4 \pm 1.2$ & $142.9 \pm 1.2$ & 0.150 & 0.983 \\
\hline $\mathrm{K}$ & $\mathrm{mEq} / \mathrm{l}$ & $4.21 \pm 0.30$ & $4.13 \pm 0.26$ & 0.395 & 0.882 \\
\hline $\mathrm{Cl}$ & $\mathrm{mEq} / \mathrm{l}$ & $104.1 \pm 1.6$ & $104.4 \pm 2.0$ & 0.658 & 0.905 \\
\hline Fasting Blood Sugar (FBS) & $\mathrm{mg} / \mathrm{dl}$ & $92.9 \pm 9.4$ & $94.3 \pm 10.3$ & 0.576 & 0.615 \\
\hline Glycohemoglobin A1C (HbA1c) & $\%$ & $4.89 \pm 0.40$ & $5.03 \pm 0.43$ & 0.001 & 0.799 \\
\hline C-Reactive Protein (CRP) & $\mathrm{mg} / \mathrm{dl}$ & $0.19 \pm 0.10$ & $0.17 \pm 0.11$ & 0.262 & 0.685 \\
\hline Lactic acid & $\mathrm{mg} / \mathrm{dl}$ & $8.17 \pm 3.45$ & $9.56 \pm 3.51$ & 0.173 & 0.471 \\
\hline Lipid peroxide (LPO) & $\mathrm{nmol} / \mathrm{ml}$ & $0.65 \pm 0.99$ & $0.32 \pm 0.08$ & 0.216 & 0.802 \\
\hline Insulin & $\mu \mathrm{U} / \mathrm{ml}$ & $9.00 \pm 7.13$ & $7.93 \pm 3.83$ & 0.543 & 0.648 \\
\hline IGF-I & $\mathrm{ng} / \mathrm{ml}$ & $197.9 \pm 68.9$ & $180.6 \pm 60.4$ & 0.007 & 0.636 \\
\hline Cortisol & $\mu \mathrm{g} / \mathrm{dl}$ & $9.9 \pm 3.0$ & $10.2 \pm 6.8$ & 0.873 & 0.562 \\
\hline $\begin{array}{l}\text { Dehydroepiandrosterone } \\
\text { Sulfate (DHEA-s) }\end{array}$ & $\mathrm{ng} / \mathrm{ml}$ & $2034.1 \pm 1136.3$ & $1928.3 \pm 899.5$ & 0.474 & 0.993 \\
\hline $\begin{array}{l}\text { 8-hydroxy-deoxyguanosine } \\
\text { (8-OHdG) generation }\end{array}$ & $\mathrm{ng} / \mathrm{kg} / \mathrm{hr}$ & $9.45 \pm 7.41$ & $8.85 \pm 4.59$ & 0.282 & 0.549 \\
\hline Isoprostane generation & $\mathrm{ng} / \mathrm{kg} / \mathrm{hr}$ & $142.2 \pm 88.7$ & $138.1 \pm 85.5$ & 0.336 & 0.427 \\
\hline 8-OHdG/CRE & $\mathrm{ng} / \mathrm{mgCRE}$ & $116.0 \pm 73.9$ & $128.5 \pm 71.7$ & 0.233 & 0.353 \\
\hline Isoprostane/CRE & $\mathrm{ng} / \mathrm{mgCRE}$ & $1846.1 \pm 869.6$ & $1837.8 \pm 1117.4$ & 0.377 & 0.338 \\
\hline
\end{tabular}

$n=17$, mean $\pm \mathrm{SD}$, paired $t$ test or Mann-Whitney $U$ test 
Table 5. Physical symptoms of Test Group

\begin{tabular}{|c|c|c|c|c|}
\hline & Before & 8 weeks later & $\begin{array}{c}P \text { value vs } \\
\text { "before" value }\end{array}$ & $\begin{array}{c}P \text { value vs } \\
\text { Control Group }\end{array}$ \\
\hline Tired eyes & $3.1 \pm 1.0$ & $2.6 \pm 1.0$ & 0.015 & 0.826 \\
\hline Blurry eyes & $2.5 \pm 0.9$ & $2.0 \pm 1.0$ & 0.035 & 0.122 \\
\hline Eye pain & $1.9 \pm 0.9$ & $1.6 \pm 0.8$ & 0.135 & 0.360 \\
\hline Stiff shoulders & $3.6 \pm 1.2$ & $3.2 \pm 1.3$ & 0.138 & 0.992 \\
\hline Muscle pain/stiffness & $3.4 \pm 1.3$ & $2.8 \pm 1.3$ & 0.017 & 0.277 \\
\hline Palpitations & $1.7 \pm 0.8$ & $1.7 \pm 0.8$ & 0.579 & 0.692 \\
\hline Dyspnea & $1.9 \pm 0.9$ & $1.7 \pm 0.8$ & 0.135 & 0.171 \\
\hline Tendency to gain weight & $3.9 \pm 0.9$ & $3.7 \pm 0.9$ & 0.361 & 0.861 \\
\hline Weight loss; thin & $1.2 \pm 0.4$ & $1.1 \pm 0.3$ & 0.331 & 0.343 \\
\hline Lethargy & $2.9 \pm 1.0$ & $2.4 \pm 1.0$ & 0.134 & 0.403 \\
\hline No feeling of good health & $2.3 \pm 1.0$ & $2.2 \pm 0.9$ & 0.528 & 0.655 \\
\hline Thirst & $1.9 \pm 0.8$ & $1.9 \pm 1.0$ & & 0.895 \\
\hline Skin problems & $2.3 \pm 1.3$ & $2.3 \pm 1.2$ & & 0.728 \\
\hline Anorexia & $1.7 \pm 0.8$ & $1.5 \pm 0.8$ & 0.331 & 0.988 \\
\hline Early satiety & $1.9 \pm 0.9$ & $1.5 \pm 0.9$ & 0.042 & 0.314 \\
\hline Epigastralgia & $1.8 \pm 0.8$ & $1.4 \pm 0.7$ & 0.010 & 0.293 \\
\hline Liable to catch colds & $2.0 \pm 1.0$ & $2.1 \pm 1.0$ & 0.842 & 0.977 \\
\hline Coughing and sputum & $2.2 \pm 1.1$ & $2.3 \pm 1.1$ & 0.528 & \\
\hline Diarrhea & $2.1 \pm 0.6$ & $2.0 \pm 0.9$ & 0.772 & 0.432 \\
\hline Constipation & $2.2 \pm 1.2$ & $2.2 \pm 1.2$ & 0.749 & 0.998 \\
\hline Headache & $2.1 \pm 0.8$ & $2.2 \pm 0.9$ & 0.790 & 0.624 \\
\hline Dizziness & $1.8 \pm 0.7$ & $1.3 \pm 0.5$ & 0.003 & 0.008 \\
\hline Tinnitus & $1.9 \pm 0.8$ & $1.7 \pm 1.0$ & 0.104 & 0.086 \\
\hline Lumbago & $3.2 \pm 1.2$ & $2.7 \pm 1.0$ & 0.088 & 0.353 \\
\hline Arthralgia & $2.7 \pm 1.0$ & $2.2 \pm 0.8$ & 0.037 & 0.257 \\
\hline Edematous & $2.6 \pm 1.2$ & $2.6 \pm 1.1$ & & 0.908 \\
\hline Easily beaking into a sweat & $3.8 \pm 1.1$ & $3.0 \pm 1.3$ & 0.026 & 0.310 \\
\hline Frequent urination & $2.4 \pm 1.1$ & $2.4 \pm 1.3$ & 0.859 & 0.727 \\
\hline Hot flashes & $1.9 \pm 1.0$ & $1.8 \pm 1.0$ & 0.834 & 0.992 \\
\hline Excessive sensitivity to cold & $2.1 \pm 1.0$ & $2.3 \pm 1.1$ & 0.260 & 0.944 \\
\hline
\end{tabular}

$n=18$, mean $\pm \mathrm{SD}$, paired $t$ test or Mann-Whitney $U$ test

count, hemoglobin content, hematocrit, GPT, $\gamma$-GTP, creatinine, and uric acid levels. Special tests such as hormone levels showed that men had significantly higher values than women in terms of cortisol $(p<0.05)$ and DHEA-s $(p<0.05)$. In oxidation stress marker tests, men showed a significantly higher $8-\mathrm{OHdG} / \mathrm{CRE}$ value than did the women. However, none of the other items showed any gender differences. Improvement rates of oxidation stress markers before and after the tests showed no gender differences.

\section{Variations associated with aging}

A correlational analysis was performed between chronological age and each test item. Regarding the results of physical measurement, height, weight, body water content and muscle volume, lean muscle mass, skeletal muscle volume and basal metabolism, they were lower with advanced age $(p<0.01)$, while body fat percentage and waist/hip ratio was higher with respect to age $(p<0.05)$. Regarding general laboratory test items, those that rose with age were creatinine $(p<0.01)$, uric acid $(p<0.05)$ and HDL-C 
Table 6. Physical symptoms of Control Group

\begin{tabular}{|c|c|c|c|c|}
\hline & Before & 8 weeks later & $\begin{array}{c}P \text { value vs } \\
\text { "before" value }\end{array}$ & $\begin{array}{l}P \text { value vs } \\
\text { Test Group }\end{array}$ \\
\hline Tired eyes & $2.7 \pm 0.8$ & $2.5 \pm 1.1$ & 0.582 & 0.826 \\
\hline Blurry eyes & $2.1 \pm 0.9$ & $2.2 \pm 0.9$ & 0.610 & 0.122 \\
\hline Eye pain & $1.7 \pm 0.7$ & $1.8 \pm 0.9$ & 0.582 & 0.360 \\
\hline Stiff shoulders & $3.6 \pm 0.9$ & $3.3 \pm 0.9$ & 0.217 & 0.992 \\
\hline Muscle pain/stiffness & $2.7 \pm 1.3$ & $2.5 \pm 1.0$ & 0.424 & 0.277 \\
\hline Palpitations & $1.7 \pm 0.8$ & $1.7 \pm 0.9$ & & 0.692 \\
\hline Dyspnea & $1.8 \pm 0.9$ & $1.9 \pm 0.9$ & 0.634 & 0.171 \\
\hline Tendency to gain weight & $3.2 \pm 1.1$ & $3.1 \pm 0.9$ & 0.499 & 0.861 \\
\hline Weight loss; thin & $1.5 \pm 0.6$ & $1.6 \pm 0.5$ & 0.334 & 0.343 \\
\hline Lethargy & $2.5 \pm 1.2$ & $2.6 \pm 1.1$ & 0.774 & 0.403 \\
\hline No feeling of good health & $2.5 \pm 0.9$ & $2.1 \pm 1.1$ & 0.207 & 0.655 \\
\hline Thirst & $2.1 \pm 1.1$ & $2.0 \pm 0.8$ & 0.634 & 0.895 \\
\hline Skin problems & $2.4 \pm 1.1$ & $2.4 \pm 1.5$ & & 0.728 \\
\hline Anorexia & $1.8 \pm 0.6$ & $1.7 \pm 0.5$ & 0.164 & 0.988 \\
\hline Early satiety & $1.9 \pm 0.8$ & $2.0 \pm 0.8$ & 0.433 & 0.314 \\
\hline Epigastralgia & $1.8 \pm 0.9$ & $1.8 \pm 0.7$ & & 0.293 \\
\hline Liable to catch colds & $1.9 \pm 0.9$ & $1.9 \pm 0.7$ & 0.751 & 0.977 \\
\hline Coughing and sputum & $1.9 \pm 0.9$ & $2.1 \pm 0.7$ & 0.104 & \\
\hline Diarrhea & $2.1 \pm 0.9$ & $2.2 \pm 0.8$ & 0.610 & 0.432 \\
\hline Constipation & $2.1 \pm 1.2$ & $2.0 \pm 1.0$ & 0.670 & 0.998 \\
\hline Headache & $2.3 \pm 1.3$ & $2.1 \pm 1.0$ & 0.262 & 0.624 \\
\hline Dizziness & $1.5 \pm 0.9$ & $1.7 \pm 0.7$ & 0.104 & 0.008 \\
\hline Tinnitus & $1.6 \pm 0.9$ & $1.8 \pm 0.9$ & 0.189 & 0.086 \\
\hline Lumbago & $2.7 \pm 1.6$ & $2.7 \pm 1.2$ & 0.774 & 0.353 \\
\hline Arthralgia & $2.0 \pm 1.2$ & $2.1 \pm 1.0$ & 0.865 & 0.257 \\
\hline Edematous & $2.3 \pm 1.0$ & $2.2 \pm 0.7$ & 0.818 & 0.908 \\
\hline Easily breaking into a sweat & $2.8 \pm 1.3$ & $2.7 \pm 1.1$ & 0.653 & 0.310 \\
\hline Frequent urination & $1.7 \pm 0.9$ & $1.8 \pm 0.7$ & 0.774 & 0.727 \\
\hline Hot flashes & $1.9 \pm 0.9$ & $1.8 \pm 0.6$ & 0.546 & 0.992 \\
\hline Excessive sensitivity to cold & $2.0 \pm 1.3$ & $2.3 \pm 1.0$ & 0.217 & 0.944 \\
\hline
\end{tabular}

$n=17$, mean $\pm \mathrm{SD}$, paired $t$ test or Mann-Whitney $U$ test

$(p<0.05)$, but $\mathrm{HbA} 1 \mathrm{c}$ decreased with age $(p<0.01)$. Tests for hormones showed that DHEA-s decreased with age $(p<0.01)$. As for oxidation stress markers, the volume of 8OHdG production decreased with age $(p<0.05)$, as did 8 OHdG/CRE $(p<0.01)$, while the improvement rates of 8 OHdG/CRE dropped with age $(p<0.05)$. Of the Anti-Aging QOL Common Questionnaire (AAQol) items, among physical symptoms, scores for "anorexia" and "excessive sensitivity to cold" increased with age $(p<0.05)$. Regarding mental symptoms, the score for "shallow sleep" increased with age $(p<0.05)$.

\section{Variations between test items}

Items in which the Test Group showed significant changes before and after the test, namely, total cholesterol, fasting blood sugar, HbAlc, lipid peroxide, "tired eyes," "blurry eyes," "muscle pain/stiffness," "early satiety," "epigastric pain," "dizziness," "arthralgia" and "easily breaking into a sweat," we studied the factors that influenced the change rates. Total cholesterol's degree of change increased in 
Table 7. Mental symptoms of Test Group

\begin{tabular}{|c|c|c|c|c|}
\hline & Before & 8 weeks later & $\begin{array}{l}P \text { value vs } \\
\text { "before" value }\end{array}$ & $\begin{array}{c}P \text { value vs } \\
\text { Control Group }\end{array}$ \\
\hline Irritability & $2.7 \pm 0.8$ & $2.6 \pm 1.1$ & 0.651 & 0.348 \\
\hline Easily angered/short-tempered & $2.6 \pm 0.9$ & $2.5 \pm 1.1$ & 0.651 & 0.131 \\
\hline Loss of motivation & $2.6 \pm 1.1$ & $2.1 \pm 1.1$ & 0.058 & 0.985 \\
\hline No feeling of happiness & $2.0 \pm 1.0$ & $2.0 \pm 1.1$ & & 0.983 \\
\hline Nothing to look forward to in life & $2.0 \pm 1.2$ & $1.8 \pm 0.9$ & 0.386 & 0.926 \\
\hline Daily life is not enjoyable & $2.1 \pm 1.0$ & $2.0 \pm 0.9$ & 0.717 & 0.800 \\
\hline Lose of confidence & $1.7 \pm 0.8$ & $1.7 \pm 0.9$ & 0.749 & 0.911 \\
\hline Reluctance to talk with others & $2.2 \pm 0.9$ & $1.7 \pm 0.8$ & 0.057 & 0.573 \\
\hline Depressed & $2.0 \pm 0.9$ & $1.9 \pm 1.0$ & 0.542 & 0.909 \\
\hline A feeling of uselessness & $1.8 \pm 0.7$ & $1.8 \pm 0.9$ & & 0.831 \\
\hline Shallow sleep & $2.2 \pm 0.9$ & $2.1 \pm 1.2$ & 0.528 & 0.998 \\
\hline Difficulty falling asleep & $1.8 \pm 0.9$ & $1.7 \pm 0.8$ & 0.269 & 0.708 \\
\hline Pessimism & $2.3 \pm 0.8$ & $2.2 \pm 1.0$ & 0.421 & 0.834 \\
\hline Lapse of memory & $2.9 \pm 0.9$ & $2.8 \pm 1.2$ & 0.430 & 0.706 \\
\hline Inability to concentrate & $2.3 \pm 1.2$ & $2.3 \pm 1.0$ & 0.790 & 0.849 \\
\hline Inability to solve problems & $2.1 \pm 1.1$ & $1.9 \pm 1.0$ & 0.163 & 0.499 \\
\hline $\begin{array}{l}\text { Inability to make judgements } \\
\text { readily }\end{array}$ & $2.1 \pm 1.0$ & $1.9 \pm 1.0$ & 0.163 & 0.315 \\
\hline $\begin{array}{l}\text { Inability to sleep because of wor- } \\
\text { ries }\end{array}$ & $1.7 \pm 0.7$ & $1.9 \pm 1.0$ & 0.331 & 0.214 \\
\hline A sense of tension & $2.1 \pm 0.9$ & $2.4 \pm 0.9$ & 0.083 & 0.065 \\
\hline $\begin{array}{l}\text { Feeling of anxiety for no special } \\
\text { reason }\end{array}$ & $1.6 \pm 0.6$ & $1.6 \pm 0.5$ & 0.717 & 0.772 \\
\hline Vague feeling of fear & $1.6 \pm 0.7$ & $1.7 \pm 0.6$ & 0.772 & 0.842 \\
\hline
\end{tabular}

$n=18$, mean $\pm \mathrm{SD}$, paired $t$ test or Mann-Whitney $U$ test

correlation to the number of cigarettes smoked $(r=0.37$, $p<0.05)$. The lower the insulin values $(r=-0.50, p<0.01)$ and $8-\mathrm{OHdG} / \mathrm{CRE}$ values $(r=-0.36, p<0.01)$, the higher the degree of change of fasting blood sugar. Women showed a high increase rate of HbAlc $(p<0.05)$. The degree of change in lipid peroxides tended to decrease in women $(p<0.01)$, in young people $(r=-0.37, p<0.01)$, in those with a high alcohol consumption $(p<0.05)$, in those with high cortisol levels $(r=-0.35, p<0.05)$, and in those with high values for height, body water content, muscle volume, lean muscle mass, skeletal muscle volume, and body fat, as well as those with high blood pressure $(r=0.46, p<0.05)$.

The improvement in scores for "tired eyes" was most marked in subjects with high cortisol values $(r=-0.38$, $p<0.05$ ), and in subjects who were extremely "irritable" $(r=-0.38, p<0.05)$ or "easily angered/short-tempered" $(r=-0.35, p<0.05)$. The degree of change in scores for "blurry eyes" was most marked in subjects with low insulin levels $(r=0.36, p<0.05)$ and low diastolic pressure $(r=0.37$, $p<0.05)$. The improvement in scores for "muscle pain/stiffness" was most marked in younger subjects $(r=0.44$, $p<0.05)$, but less so for subjects with "shallow sleep" $(r=0.43, p<0.05)$. Scores for "early satiety" $(r=0.42$, $p<0.05)$ and "epigastric pain" $(r=0.51, p<0.01)$ tended to improve less readily in subjects with "shallow sleep." There were no factors that affected the improvement rates in the score for "dizziness." The improvement in score for "arthralgia" was most marked in heavy smokers $(r=-0.36$, $p<0.05)$, subjects who were "irritable" $(r=-0.39, p<0.05)$, "easily angered/short-tempered" $(r=-0.35, p<0.05)$, or "depressed" $(r=-0.38, p<0.05)$. The scores tended to improve less in those who engaged in regular and vigorous exercise $(r=0.53, p<0.05)$. The degree of improvement in the scores for "easily breaking into a sweat" tended to be low in subjects with high cortisol values $(r=-0.36, p<0.05)$, but was higher in subjects who had a "loss of motivation" 
Table 8. Mental symptoms of Control Group

\begin{tabular}{|c|c|c|c|c|}
\hline & Before & 8 weeks later & $\begin{array}{l}P \text { value vs } \\
\text { "before" value }\end{array}$ & $\begin{array}{l}P \text { value vs } \\
\text { Test Group }\end{array}$ \\
\hline Irritability & $2.3 \pm 1.0$ & $2.5 \pm 0.6$ & 0.433 & 0.348 \\
\hline Easily angered/short-tempered & $2.3 \pm 1.1$ & $2.5 \pm 0.6$ & 0.384 & 0.131 \\
\hline Loss of motivation & $2.3 \pm 0.8$ & $2.0 \pm 0.7$ & 0.055 & 0.985 \\
\hline No feeling of happiness & $2.2 \pm 0.8$ & $2.1 \pm 0.7$ & 0.774 & 0.983 \\
\hline Nothing to look forward to in life & $2.1 \pm 0.9$ & $1.9 \pm 0.5$ & 0.499 & 0.926 \\
\hline Daily life is not enjoyable & $2.3 \pm 0.8$ & $2.0 \pm 0.5$ & 0.217 & 0.800 \\
\hline Lose of confidence & $2.3 \pm 0.8$ & $1.9 \pm 0.5$ & 0.173 & 0.911 \\
\hline Reluctance to talk with others & $1.9 \pm 0.8$ & $1.9 \pm 0.7$ & & 0.573 \\
\hline Depressed & $2.1 \pm 0.8$ & $2.1 \pm 0.7$ & 0.774 & 0.909 \\
\hline A feeling of uselessness & $2.1 \pm 0.5$ & $2.1 \pm 0.5$ & & 0.831 \\
\hline Shallow sleep & $2.5 \pm 1.1$ & $2.3 \pm 0.9$ & 0.189 & 0.998 \\
\hline Difficulty falling asleep & $2.3 \pm 1.0$ & $1.9 \pm 0.7$ & 0.019 & 0.708 \\
\hline Pessimism & $2.6 \pm 1.0$ & $2.2 \pm 0.7$ & 0.054 & 0.834 \\
\hline Lapse of memory & $2.9 \pm 0.7$ & $2.9 \pm 0.6$ & & 0.706 \\
\hline Inability to concentrate & $2.2 \pm 0.7$ & $2.3 \pm 0.6$ & 0.582 & 0.849 \\
\hline Inability to solve problems & $2.1 \pm 0.5$ & $2.2 \pm 0.7$ & 0.334 & 0.499 \\
\hline $\begin{array}{l}\text { Inability to make judgements } \\
\text { readily }\end{array}$ & $2.2 \pm 0.8$ & $2.3 \pm 0.6$ & 0.774 & 0.315 \\
\hline $\begin{array}{l}\text { Inability to sleep because } \\
\text { of worries }\end{array}$ & $2.4 \pm 1.0$ & $2.1 \pm 0.8$ & 0.136 & 0.214 \\
\hline A sense of tension & $2.4 \pm 0.6$ & $2.3 \pm 0.7$ & 0.433 & 0.065 \\
\hline $\begin{array}{l}\text { Feeling of anxiety for no special } \\
\text { reason }\end{array}$ & $1.9 \pm 0.9$ & $1.8 \pm 0.6$ & 0.499 & 0.772 \\
\hline Vague feeling of fear & $1.7 \pm 0.6$ & $1.7 \pm 0.7$ & & 0.842 \\
\hline
\end{tabular}

$n=17$, mean $\pm \mathrm{SD}$, paired $t$ test or Mann-Whitney $U$ test

$(r=0.41, p<0.05)$

\section{Discussion}

\section{Amino acids}

A healthy individual with a standard weight of $60 \mathrm{~kg}$ is said to require about 2,000 to $2,400 \mathrm{kcal}$ per day, with the ratio among carbohydrates, protein and fat ideally being $6: 2: 2$. The ideal daily protein intake, moreover, is said to be within the range of 80 to $120 \mathrm{~g}$. We await more investigations on the optimal doses based on EBM. It is recommended that between 5 and $10 \mathrm{~g}$ of amino acids be replenished in the form of amino acid supplements if individuals fail to meet the required protein intake level due to eating irregularly, or if their masticatory capabilities have weakened [1].

In the course of protein being synthesized from amino acids in vivo, it requires the aid of vitamin $\mathrm{B}_{6}$ and other substances. Antioxidants such as vitamin $\mathrm{C}$ and $\mathrm{E}$ are also useful in suppressing the free radicals that develop during this process.

The human body is composed of 20 types of amino acids. There are ten amino acids that cannot be synthesized in vivo and therefore must be obtainable from food: valine, leucine, isoleucine, histidine, phenylalanine, tryptophan, lysine, methionine, arginine, and threonine. There are ten nonessential amino acids that can be synthesized in vivo: glutamine, proline, glycine, asparagine, glutamic acid, aspartic acid, cysteine, tyrosine, serine, and alanine.

The synthetic capability in vivo of nonessential amino acids generally declines with age. What is especially important is not chronological age, but hormonal age, as revealed in a reduction in DHEA, growth hormones and IGF-I. Growth hormones and IGF-I directly affect amino acid synthesis, and decreased DHEA that is synthesized in the adrenal gland indirectly affects the synthesis of amino acids 
and proteins via reductions in protein-synthesizing hormones that are synthesized based on DHEA and in androgens that have protein anabolic activity.

\section{Carnitine}

Carnitine is a type of amino acid. L-carnitine works to combine with acyl-CoA that is generated intracellularly from free fatty cells to produce acylcarnitine and to enable the passage of acyl-CoA through endosporium of mitochondria [8-13]. Therefore, it is essential to the metabolism of intracellular fat; it also works to reduce the dependence of the body on sugar as a source of energy. It protects against degeneration of nerve cells, so it is believed to be useful for preventing loss of short-term memory, Alzheimer's disease, and depression [11]. There are also reports that carnitine helps in the treatment of liver and heart diseases [13], of male reproductive functions and mental stress [10] and in restoring visual acuity. It also has the potential to reduce the levels of lipids circulating within the blood and thus lower the risk of arteriosclerosis [13]. Deficiency in carnitine induces impaired intracellular fat metabolism [8]. Foods that contain abundant carnitine include mutton and beef.

A study that measured the amount of carnitine in the plasma and the liver of 68 pediatric chronic liver disease and 36 cirrhosis patients showed that the latter patients had low plasma carnitine concentrations [14]. Meanwhile, in a study using 382 hyperlipidemia patients taking statin drugs (167 males and 215 females; mean age: 63.17 years), patients given oral statins had higher CPK levels than normal populations, and had significantly lower plasma acylcarnitine levels. This suggests the possibility that, for some unknown reason, individuals on oral statin drugs have fatty acid metabolic abnormalities due to abnormal carnitine metabolism, and that this may be one of the causes of the rise in CPK levels and myopathy [15].

\section{Carnitine in animal models}

Carnitine influences metabolic mechanisms in numerous ways. A study in which $200 \mathrm{mg} / \mathrm{kg}$ of L-carnitine was administered for 4 weeks to streptomycin-induced diabetic rats showed that the drug helped to restore serum IGF-I to close to normal concentrations [16]. In septic rats that had undergone appendix ligation and perforation, the concentration of endotoxins in the blood rose, while the concentrations of branched chain amino acids in the blood (leucine, isoleucine and valine) as well as glutamine were lower. Administration of L-carnitine, however, is shown to suppress declines in the concentrations of branched-chain amino acids and glutamine [17]. Aged rats, in particular, show decreases in the content of protein in each tissue as well as the level of protein-bound hexose, hexosamine, sialic acid, and fucose. However, intra-abdominal administration of L-carnitine is shown to improve the conditions of these glycoproteins. On the other hand, in young rats, the administration of L-carnitine does not induce these changes, suggesting that carnitine helps in normalizing age-induced changes in glycoproteins [18].

Carnitine also affects the gastrointestinal tract. There is a reduction in gastric mucins and prostaglandin $\mathrm{E}_{2}$ of rats subjected to cold stress. However, the administration of L-carnitine suppresses this reduction as well as the development of gastric mucosal lesions and formation of lipid peroxide. Administration of L-carnitine is also shown to elevate catalase activity in the blood and inside the gastric mucosal membrane [19].

Carnitine also acts on the sensory organs. In a study in which palmitoyl-DL-carnitine $\left(1 \times 10^{-4} \mathrm{M}\right)$ was administered to guinea pigs via perilymphatic reflux, measurements of the endocochlear DC potential and cochlear microphonics showed the former to have dropped to $+8 \sim+16 \mathrm{mV}$, and the latter to $30 \sim 39 \%$, suggesting a direct effect of palmitoyl-DL-carnitine on cochlear hair cells [20].

Several reports have been published on the effects of carnitine on the heart. Administration of adriamycin reduces free carnitine in rat cardiac muscle and boosts acylcarnitine levels. These, however, were shown to improve after 500 $\mathrm{mg} / \mathrm{kg}$ of L-carnitine was administered intra-abdominally on a daily basis, showing that L-carnitine counteracts cardiotoxicity of adriamycin [21]. In a study using rat models of ischemic myocardial disorders, administration of L-carnitine prior to re-reflux was shown to increase free carnitine, activating fatty acid metabolism after re-reflux and improving glycolytic metabolism that was impaired because of ischemia, thereby improving myocardial metabolism and promoting the recovery of myocardial function [22]. In another study that investigated the effects of Lcarnitine in which extracted reflux heart of diabetic rats (a state of diabetic cardiac dysfunction such as reduction in systolic and diastolic functions) was used, reduced intramyocardial carnitine content was seen to improve with oral administration of propionyl-L-carnitine. The drug also increased intra-myocardial carnitine and improved cardiac function [23].

\section{Clinical effects of carnitine}

Initially, reports on the activity of carnitine in humans mostly focused on cardiac function. In a study in which $200 \mathrm{mg} /$ day of L-carnitine was administered to nine patients with heart disease (i.e., history of myocardial infarction) after undergoing ergometer exercise tests, the subjects showed improvements in exercise time peak value and maximum oxygen consumption volume, as well as higher lactic acid and ventilation thresholds. L-carnitine was therefore shown to improve loss of exercise tolerance [24]. In a study in which L-carnitine was administered orally for 12 weeks, exercise tolerance significantly improved in exer- 
tional angina patients, while 5 out of 9 chronic congestive heart failure patients were switched to a less severe NYHA class category, and 6 patients saw their systemic conditions improve [25]. In a study in which $60 \mathrm{mg} / \mathrm{kg}$ of levocarnitine was administered via intravenous drip to 15 ischemic heart disease patients, myocardial metabolism and blood flow increase during exercise were stepped up, indicating that the drug demonstrated anti-angina activity [26]. In a study that compared the effects of levocarnitine chloride and a placebo in 46 stable, exertional angina patients, administration of levocarnitine was shown to extend the time of exercise in multiple-stage treadmill exercise tests and prolong the time to onset of angina pain [27]. Meanwhile, a multi-center, double-blind, placebo-controlled intra-group comparative test was performed, targeting 114 angina patients who still suffered angina pain despite administration of timed-released nitric acid drugs. A combination of timed-released nitric acid drugs and levocarnitine chloride was used. As a result, compared with the Control Group, the Levocarnitine Group saw significant improvements in their exercise ECG [28]. Levocarnitine preparations are used today as L-Cartin ${ }^{\circledR}$, a drug covered by Japanese health insurance.

There are also reports on the use of L-carnitine as a dietary supplement for patients with renal failure. In a study of 17 hemodialysis patients who were undergoing continuous maintenance therapy comprising erythropoietin, the maintenance dosage of erythropoietin correlated negatively to serum free carnitine concentration. Twenty weeks after taking $500 \mathrm{mg}$ /day of carnitine chloride, four patients saw an over $20 \%$ improvement in their hematocrit values, three of whom were able to reduce the dose of erythropoietin [29]. In a study targeting 7 chronic renal failure patients undergoing hemodialysis (mean: $61 \pm 17$ years, mean history of dialysis: $17 \pm 10$ years), as a result of administration of L-carnitine $(1,000 \mathrm{mg} /$ day for the first month and $500 \mathrm{mg} / 1-2$ days after the second month; the drug was continued for 2 or more years), serum total carnitine concentration improved, the dose of erythropoietin could be decreased, and palpitations as well as post-dialysis fatigue and lethargy improved. These results suggest that dietary supplementation with L-carnitine is useful for improving the QOL of dialysis patients [30].

Recently, reports related to supplements containing carnitine have also been published. In an open test in which ten healthy individuals ( 4 males; mean age, $40.0 \pm 4.2$ years; BMI, 27.2 \pm 2.2 ; 6 females; mean age, $35.2 \pm 9.4$ years; BMI, $26.9 \pm 2.8$ ) took Metabolic L-Carnitine Plus ${ }^{\circledR}$ for 40 days, both men and women saw a significant reduction in weight, BMI, body fat percentage, bust, waist, hip, upper arm, thigh, and calf circumference; a significant rise in total carnitine, free carnitine, and acylcarnitine; and a significant drop in total cholesterol, triglycerides, blood sugar and lactic acid. The results show the potential usefulness of supple- ments containing carnitine in promoting lipolysis and energy production in obese individuals [31].

\section{Garcinia cambogia extract}

Hydroxycitric acid is a major acid component of the tropical plants Garcinia cambogia [32]. (2S, 3S)-HCA from G. cambogia was shown to be a potent inhibitor of ATP citrate lyase, which catalyzes the extramitochondrial cleavage of citrate to oxaloacetate and acetyl-CoA. HCA used in dietary weight loss supplement. In the present study, $500 \mathrm{mg} /$ day of hydroxycitric acid had no significant effect on the body component. The dietary efficacy was not indicated.

\section{Results of our tests}

Results of the analysis of the Common Questionnaire (AAQol) showed that the Test Group revealed significant improvements in a number of physical symptoms. This is believed to be attributable to the comprehensive actions of amino acids and multiple vitamins contained in the Product. Improvement effects on "dizziness," in particular, showed significant differences between the Test Group and the Control Group, indicating that the Product significantly improved "dizziness." This is also believed to be related to the direct activity of carnitine on cochlear hair cells [20]. Among the Test Group, symptoms such as "early satiety" and "epigastric pain" improved significantly. Carnitine is known to improve digestive tract movements and to suppress lesions in the gastric mucosal membrane [19], so it is believed to be involved in improving both "early satiety" and "epigastric pain."

Regarding ocular symptoms, a number of reports have been released. One states that there was a reduction in carnitine inside the ocular lens of diabetic rats [33], while another found carnitine to be effective for ischemic re-reflux disorder in the retina of guinea pigs [34]. There is also a report of a clinical test with human subjects that showed a supplement containing carnitine to counter macular degeneration [35]. The fact that ocular symptoms such as "tired eyes" and "blurry eyes" significantly improved in the Test Group may be attributable to this recovery of the concentration of carnitine in the ocular tissue.

In our laboratory tests, a significant rise was seen in total cholesterol, fasting blood sugar, and HbAlc. Although past reports have shown that carnitine corrects lipid and sugar metabolism $[8,10,13]$, our test produced a different result, showing that "early satiety" and "epigastric pain" were alleviated, so it is highly likely that other elements may have come into play, such as increased appetite and improved caloric intake from food. As for oxidation stress markers, lipid peroxides decreased significantly in the Test Group. Carnitine plays an important role in lipid metabolism [8] and possesses anti-oxidation activity $[19,22,34,36]$, so a 
decrease in lipid peroxides is not contradictory.

These findings suggest that the consumption of the supplement can reduce the oxidative damage and some symptoms in the human; however, the effect on QOL was equivocal from the results of the effect on lipid metabolism. Garcinia cambogia extract did not show dietary efficacy.

\section{Muscular fatigue}

If a person undergoes muscular load training for $15 \mathrm{~min}$ utes, lactic acid is produced in the muscular tissues; $30 \mathrm{~min}$ utes later, growth hormone-reactive secretion peaks from the pituitary gland. Two hours after the start of exercise, fat breaks down, and the concentration of free fatty acid in the blood peaks [2]. In individuals engaging in aerobic exercises such as jogging and aerobics, glucose and fatty acids enter the TCA circuit, which is one of the body's energyproducing systems, to generate energy. In contrast, in anaerobic exercises, such as weightlifting, lactic acid is generated without having glucose and fatty acids enter the TCA circuit. Although lactic acid is a factor that encourages the secretion stimulation of growth hormones, it can also cause muscular pain and fatigue.

In our test, scores for "muscle pain/stiffness" changed from $3.4 \pm 1.3$ to $2.8 \pm 1.3(-17.6 \%, p=0.017)$ in the Test Group, and from $2.7 \pm 1.3$ to $2.5 \pm 1.0(-7.4 \%)$ in the Control Group, showing no significant differences in the improvement rate between the two groups. However, it improved significantly in the Test Group only. An important activity of carnitine is that it combines with acyl-CoA, which is produced from free fatty acids inside the cells, to enable the passage of acyl-CoA through the mitochondrial endosporium [8-13]. This is most likely because, even in muscle cells, which are packed with mitochondria, replenishing carnitine improves the status of metabolism to bring about favorable effects.

\section{Correlations between test items}

Administration of the Product induced significant changes in total cholesterol, fasting blood sugar, HbAlc, and lipid peroxide, as well as symptoms such as "tired eyes," "blurry eyes," "muscle pain/stiffness," "early satiety," "epigastric pain," "dizziness," "arthralgia," and "easily breaking into a sweat."

The fact that total cholesterol increases in proportion to the number of cigarettes smoked implies that total cholesterol and smoking, which are risk factors for arteriosclerosis, may work synergistically. This reconfirms the importance of reducing these risk factors one by one to prevent diseases related to arteriosclerosis such as cerebral apoplexy and heart disease. We were not able to identify the relationship between the degree of change in fasting blood sugar and insulin values and $8-\mathrm{OHdG} / \mathrm{CRE}$.

Lipid peroxides are believed to be relatively high in women who have high body fat percentages, in young people who drink heavily, and in individuals with high values for height, body water content, muscle volume, lean muscle mass, and skeletal muscle volume; these values are liable to drop because of antioxidative substances contained in the Product. It appears, however, that elderly individuals do not demonstrate antioxidative effects very readily. The relationship with cortisol is not clear.

Individuals who have high cortisol values, and who show extreme "irritability" or who are "easily angered/shorttempered" are believed to suffer extensive "tired eyes." It may be that it was relatively easy to see their symptoms related to "tired eyes" improve because of the Product. The causal relationship between the degree of change in the scores for "blurry eyes," insulin value and diastolic blood pressure is not clear. It appears that the older the individual, the more difficult for his or her "muscle pain/stiffness" to improve; the more a person suffers "shallow sleep," the more difficult for it to improve because of insufficient tissue restoration during sleep. It appears that symptoms of "early satiety" and "epigastralgia" are difficult to improve in individuals who often experience "shallow sleep." This suggests the importance of sleep. Heavy smokers and people who have major symptoms of "irritability", "easily angered/ short-tempered," and being depressed tend to manifest symptoms of "arthralgia" more strongly than others, so it may be that these symptoms tend to improve more readily as a result of using the Product. People who exercise extensively to begin with have their "arthralgia" already in a steady state, so it may be that their symptoms do not change very readily, even as a result of using the Product. We were unable to identify any relationship between "easily breaking into a sweat" and cortisol value or "loss of motivation."

\section{Acknowledgement}

Research for the project was supported by Antiaging Bank Inc. Tokyo, Japan (www.yonei-labo.com), Gingakobo Inc., Nagoya, Japan (www.gingakobo.jp) and the Wellness Research Institute, Osaka, Japan (www.natural-life.jp).

\section{Abbreviations}

QOL, Quality of Life; AAQol, Anti-Aging QOL Common Questionnaire; 8-OHdG, urinary 8-hydroxydeoxyguanosine; CRE, creatinine; BMI, Body Mass Index; RBC, Red Blood Cells; WBC, White Blood Cells; HGB, hemoglobin content; HCT, hematocrit; GOT, glutamateoxaloacetate transaminase; GPT, glutamate-pyruvate transaminase; $\gamma$-GTP, $\gamma$-glutamyl transpeptase; ALP, alkaline phosphatase; CPK, creatinine phosphokinase; CRP, C-reactive protein; BUN, blood urea nitrogen; $\mathrm{Cr}$, blood creatinine; UA, uric acid; T-Chol, total cholesterol; HDL-C, HDL- 
cholesterol; LDL-C, LDL-cholesterol; TG, triglycerides; FBS, fasting blood sugar; $\mathrm{Hb} \mathrm{A} 1 \mathrm{C}$, glycohemoglobin A1C; DHEA-s, dehydroepiandrosterone sulfate; IGF-I, insulinlike growth factor; LPO, lipid peroxides; MCV, Mean Corpuscular Volume; MCH, Mean Corpuscular Hemoglobin.

\section{References}

[1] Yonei, Y. and Mizuno, Y.: The human dock of tomorrowAnnual health checkup for anti-aging. Ningen Dock, 19, 5-8, 2005.

[2] Yonei, Y., Mizuno, Y., Togari, H., and Sato, Y.: Muscular resistance training using applied pressure and its effects on the promotion of growth hormone secretion. Anti-Aging Medical Research, 1, 13-27, 2004.

[3] Yonei, Y., Mizuno, Y., and Katagiri, E.: Effects of cosmetics therapy using isoflavone and pine bark extract on the skin and QOL: A double-blind placebo-controlled trial. AntiAging Medical Research, 1, 48-58, 2004.

[4] Yonei, Y., Iwaita, Y., Muramatsu, K., and Mizuno, Y.: The anti-aging secrets of Japanese executives. Anti-Aging Medical Research, 2, 61-69, 2005.

[5] Thompson, H.J., Heimendinger, J., Gillette, C., Sedlacek, S.M., Haegele, A., O'neill, C., and Wolfe, P.: In vivo investigation of changes in biomarkers of oxidative stress induced by plant food rich diets. J. Agric. Food Chem., 53, 61266132, 2005.

[6] Urso, M.L. and Clarkson, P.M.: Oxidative stress, exercise, and antioxidant supplementation. Toxicology, 189, 41-54, 2003.

[7] Piconi, L., Quagliaro, L., and Ceriello, A.: Oxidative stress in diabetes. Clin. Chem. Lab. Med., 41, 1144-1149, 2003.

[8] Reda, E., D’Iddio, S., Nicolai, R., Benatti, P., and Calvani, M.: The carnitine system and body composition. Acta Diabetol., 40 Suppl 1, S106-113, 2003.

[9] Sweeney, J.D. and Arduini, A.: L-carnitine and its possible role in red cell and platelet storage. Transfus. Med. Rev., 18, 58-65, 2004.

[10] Alesci, S., De Martino, M.U., Kino, T., and Ilias, I.: LCarnitine is a modulator of the glucocorticoid receptor alpha. Ann. NY Acad. Sci., 1024, 147-152, 2004.

[11] Virmani, A. and Binienda, Z.: Role of carnitine esters in brain neuropathology. Mol. Aspects Med., 25, 533-549, 2004.

[12] Saper, R.B., Eisenberg, D.M., and Phillips, R.S.: Common dietary supplements for weight loss. Am. Fam. Physician, 70, 1731-1738, 2004.

[13] Ferrari, R., Merli, E., Cicchitelli, G., Mele, D., Fucili, A., and Ceconi, C.: Therapeutic Effects of 1-Carnitine and Propionyl1-carnitine on Cardiovascular Diseases: A Review. Ann. NY Acad. Sci., 1033, 79-91, 2004.

[14] Selimoglu, M., Aydogdu, S., Yagci, R.V., and Huseyinov, A.: Plasma and liver carnitine status of children with chronic liver disease and cirrhosis. Pediatrics International, 43, 391395, 2001.

[15] Eto, S., Ogawara, S., Kaneoka, H., Tamura, K., Hirose, S.,
Itoguchi, H., and Saito, T.: Patient Administration of HMGCoA reductase inhibitor and rising myogenic enzyme levels. Therapeutic Research, 23, 1340-1343, 2002 (in Japanese).

[16] Heo, Y-R., Kang, C-W., and Cha, Y-S.: L-Carnitine Changes the Levels of Insulin-like Growth Factors (IGFs) and IGF Binding Proteins in Streptozotocin-induced Diabetic Rat. J. Nutri. Sci. Vitaminol., 47, 329-334, 2001.

[17] Hayashi, N., Yoshihara, D., Kashiwabara, N., Takeshita, Y., Handa, H., and Yamakawa, M.: Effect of carnitine on decrease of branched chain amino acids and glutamine in serum of septic rats. Biol. Pharm. Bull., 19, 157-159, 1996.

[18] Palaniyappan, A. and Chinnakkannu, P.: Replenishment of Lcarnitine to aged rats and its effect on glycoprotein. J. Clin. Biochem. Nutri., 26, 193-200, 1999.

[19] Izguet-Uysal, V.N., Agac, A., and Derin, N.: Effect of carnitine on stress-induced lipid peroxidation in rat gastric mucosa. J. Gastroenterol., 36, 231-236, 2001.

[20] Yoshitoshi, Y.: The effect of Palmitoyl Carnitine on the electrical phenomenon of the cochlea. Kawasaki Igakukaishi, 19, 91-101, 1993 (in Japanese).

[21] Takada, O.: Early stage, Post Administration of Adrianmycin and movement of carnitine in the myocardium. Nihon Shouni Junkanki Gakkai Zasshi, 9, 800-808, 1994 (in Japanese).

[22] Yamamoto, M., Lee, J., and Shimizu, H.: Improvement in myocardial metabolism by L-carnitine in the reperfusion rabbit model (making use of 1H-MRS). Junkankika, 35, 560563, 1994 (in Japanese).

[23] Terada, T., Matsubara, T., and Ikoma, Y.: The effect of carnitine on diabetic heart function. Shinkin Kouzou Taishya, 14, 95-99, 1992 (in Japanese).

[24] Watanabe, S., Ajisaka, R., Masuoka, T., Yamanouchi, T., Saitou, T., Toyama, M., Takeyasu, N., Sakamoto, K., and Sugishita, Y.: Effects of L- and DL-carnitine on patients with impaired exercise tolerance. Jpn. Heart J., 36, 319-331, 1995.

[25] Kobayashi, A., Masumura, Y., and Yamazaki, N.: L-carnitine treatment for congestive heart failure-experimental and clinical study. Jpn. Circ. J., 56, 86-94, 1992.

[26] Fujiwara, M., Nakano, T., Tamoto, S., Yamada, Y., Fukai, M., Takada, K., Ashida, H., Shimada, T., Ishihara, T., and Seki, I.: Effect of L-carnitine in patients with ischemic heart disease. Journal of Cardiology, 21, 493-504, 1991 (in Japanese).

[27] Sotohata, I., Noda, S., Hayashi, H., Ito, T., and Mizuno, Y.: Clinical assessment of Levocarnitine chloride (LC-80) administered to angina pectoris patients subjected to treadmill exercise physical tolerance test. Rinshou Yakurigaku (Clinical Pharmacology), 20, 607-618, 1989 (in Japanese).

[28] Yoshitoshi, Y., Yamazaki, N., and Imura, K.: Clinical trial of a multicenter double-blind intergroup comparison on the efficacy of Levocarnitine chloride (LC-80) on the pathology of Angina pectoris. Yakuri To Chiryou, 17, 5015-5035, 1989 (in Japanese).

[29] Endo, S. and Yamamoto, Y.: Study of carnitine replacement therapy for the maintenance of erythropoietin levels in the hemodialysis patient. Ketsueki Frontier, 9, 59-64, 1998 (in Japanese). 
[30] Yamada, M., Niikura, H., Yanagisawa, K., Sone, H., and Shirota, T.: L-carnitine for the dialysis patient. Nagano-ken Touseki Kenkyukaishi, 25, 46-47, 2002 (in Japanese).

[31] Sugita, T., Saito, Y., Hatano, T., Koizumi, Y., and Kara, T.: Efficacy and safety of metabolic L-Carnitine Plus Diet. Shinyaku To Rinsho, 53, 191-201, 2004.

[32] Hida, H., Yamada, T., and Yamada, Y.: Production of hydroxycitric acid by microorganisms. Biosci. Biotechnol. Biochem., 69, 1555-1561, 2005.

[33] Pessotto, P., Liberati, R., Petrella, O., Romanelli, L., Calvani, M., and Peluso, G.: In experimental diabetes the decrease in the eye of lens carnitine levels is an early important and selective event. Exp. Eye. Res., 64, 195-201, 1997.

[34] Alagoz, G., Celiker, U., Ilhan, N., Yekeler, H., Demir, T., and Celiker, H.: L-carnitine in experimental retinal ischemiareperfusion injury. Ophthalmologica, 216, 144-150, 2002.

[35] Feher, J., Papale, A., Mannino, G., Gualdi, L., and Balacco Gabrieli, C.: Mitotropic compounds for the treatment of agerelated macular degeneration. The metabolic approach and a pilot study. Ophthalmologica, 217, 351-357, 2003.

[36] Arafa, H.M.: Sayed-Ahmed MM. Protective role of carnitine esters against alcohol-induced gastric lesions in rats. Pharmacol. Res., 48, 285-290, 2003. 